\title{
High energy protons from PKS 1333-33
}

\author{
L. A. Anchordoqui ${ }^{a}$, G. E. Romero ${ }^{a, b}$, S. E. Perez Bergliaffa ${ }^{c}$ and J. A. Combi ${ }^{b}$ \\ ${ }^{a}$ Departamento de Física, UNLP, C.C. 67, (1900) La Plata Argentina \\ ${ }^{b}$ Instituto Argentino de Radioastronomía, C.C. 5, (1894) Villa Elisa, Argentina \\ ${ }^{c}$ LAFEX/CBPF-Centro Brasileiro de Pesquisas Fisicas, Rua Xavier Sigaud 150, Rio de Janeiro, 22290-RJ, Brazil
}

In this letter we give an account of the possible acceleration of protons in the outer radio lobes of the active galaxy PKS 1333-33. We also make estimates of the arrival energy spectrum. PACS number(s): 96.40 - 98.70.S - 95.85.R - 13.85.T

Radio galaxies considered as sources of cosmic rays (CR) beyond the Greisen-Zatsepin-Kuz'min cutoff in must be quite close to our own galaxy $(z \leq 0.03)$. There are just a few objects within this range and, consequently, the expected ultra high energy $\mathrm{CR}$ distribution on the sky should be highly anisotropic. Assuming that the intergalactic magnetic field is near $1 \mathrm{nG}$, it should be expected an excess of $\mathrm{CR}$ detections at energies larger than $5 \times 10^{19} \mathrm{eV}$ within regions of angular radius $\theta \leq 15^{\circ}$ and centered at the positions of the nearest active galaxies [2]. According to this picture, the southern CR-sky should be dominated by three outstanding sources: Cen A (the nearest radio galaxy) which would provide the most energetic particles detectable on Earth [3], Pictor A (a strong source with a flat radio spectrum) which would contribute with the larger CR flux [4], and PKS 1333-33 which might be a source of events similar to those recently detected in the Northern Hemisphere. In addition to these sources, there are other two southern candidates, Fornax A $(z=0.057)$ and PKS 2152-69 $(z=0.027)$, which could provide contributions to the CR flux above the cutoff. An approximate theoretical picture of the up to now unexplored ultra high energy CR southern sky is consequently at our disposal. The present letter is devoted to $\mathrm{CR}$ production in the outer region of the nearby southern active galaxy PKS 1333-33 and the spectral modifications arisen from their propagation through intergalactic space.

The radio galaxy PKS 1333-33 is made up of a core, two symmetric jets, and two extended radio lobes. Fig. 1 shows the entire source at $20 \mathrm{~cm}$ according to the VLA map obtained by Killeen et al. [5]. The core has been identified with the E1 galaxy IC 4296, which has a redshift $z=0.013 \pi 6$. The distance to the source is $35.2 h^{-1}$ Mpc, $\left(h=H_{0} / 100 \mathrm{~km}^{-1} \mathrm{~s} \mathrm{Mpc}\right.$, hereafter we shall adopt $h=0.65[7])$. The large-scale structure of PKS 1333-33 has been studied in detail at 1.3, 2, 6, and $20 \mathrm{~cm}$ with an angular resolution of 3.2" [5]. The jets are slightly bent, presumably as a consequence of the motion of the core with respect to the intergalactic medium. The total flux density of the source at $20 \mathrm{~cm}$ is $\sim 14.5 \mathrm{Jy}$. The integrated radio luminosity is $\sim 2.5 \times 10^{41} \mathrm{erg} \mathrm{s}^{-1}$ assuming a spectral index of $\alpha=0.6$ and frequency cutoffs at $10^{7}$ and $10^{11} \mathrm{~Hz}$. Actually, the value $\alpha=0.6$ is correct only for the extended radio lobes. The spectral index steep- ens in the jets, reaching $\alpha=1$, while the core has flatter values: $\alpha \sim 0.3$.

The hot spots of extended radio sources are regions of strong synchrotron emission [8]. These regions are produced when the bulk kinetic energy of the jets ejected by a central active source (supermassive black hole plus accretion disk) is reconverted into relativistic particles and turbulent fields at a "working surface" in the head of the jets [9]. The speed $v_{\mathrm{h}}$ with which the head of a jet advances into the intergalactic medium of particle density $n_{\mathrm{e}}$ can be obtained by balancing the momentum flux in the jet against the momentum flux of the surrounding medium. Measured in the frame comoving with the advancing head, $v_{\mathrm{h}} \approx v_{\mathrm{j}}\left[1+\left(n_{\mathrm{e}} / n_{\mathrm{j}}\right)^{1 / 2}\right]^{-1}$, where $n_{\mathrm{j}}$ and $v_{\mathrm{j}}$ are the particle density and the velocity of the jet flow, respectively. Clearly, $v_{\mathrm{j}}>v_{\mathrm{h}}$ for $n_{\mathrm{e}} \geq n_{\mathrm{j}}$, in such a way that the jet will decelerate. The result is the formation of a strong collisionless shock, which is responsible for particle reacceleration and magnetic field amplification. The acceleration of particles up to ultrarelativistic energies in the hot spots is the result of repeated scattering back and forth across the shock front. The particle deflection in this mechanism is produced by Alfvén waves in the turbulent magnetic field. Biermann and Strittmatter 110 have studied this process assuming that the energy density per unit of wave number of MHD turbulence is of Kolmogorov type. According to their calculations, the highest energy of protons injected in the intergalactic medium from the hot spot can be obtained by balancing the gains and losses in the diffusive shock acceleration process:

$$
\begin{aligned}
E_{\max } & =7.8 \times 10^{5} \beta_{\mathrm{jet}}^{3 / 2} u^{3 / 4} R_{\mathrm{hs}}^{-1 / 2} \\
& \times B_{-5}^{-5 / 4}(1+A a)^{-3 / 4} \mathrm{EeV}
\end{aligned}
$$

where $\beta_{\text {jet }}$ stands for the jet velocity in units of $c, u$ is the ratio of turbulent to ambient magnetic energy density in the hot spot (of radius $R_{\mathrm{hs}}$ measured in kpc), $B_{-5}$ is the magnetic field in units of $10^{-5} \mathrm{G}, a$ is the ratio of photon to magnetic energy density, and $A$ gives a relative strength of $\gamma p$ interactions against the synchrotron emission.

An interesting feature of PKS 1333-33 is an intense region of synchrotron emission localized at the outer edge of the eastern lobe. This region can be considered as a 


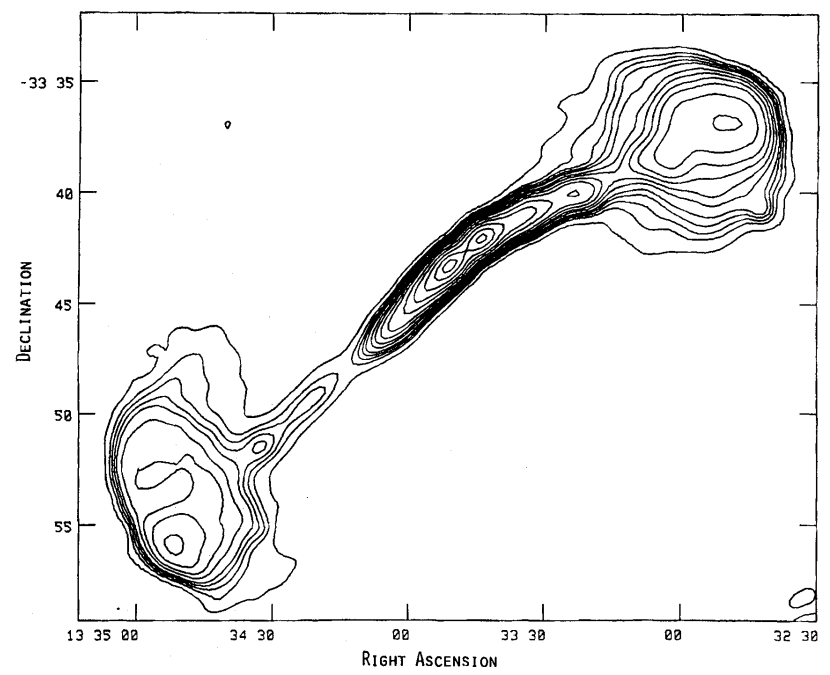

FIG. 1. VLA image of the entire PKS 1333-33 source obtained at a wavelength of $20 \mathrm{~cm}$ by Killeen et al.

"working surface" formed by the deceleration of the jet. This interpretation is supported by VLA polarimetric observations, which show a change in the field orientation from parallel to perpendicular to the jet axis [5]. This change is probably due to the rearrangement of the field lines in the post-shock region. The degree of linear polarization in the eastern lobe is in the range $20 \%-40 \%$. The synchrotron parameters estimated for this region are: the minimum energy density $\left(\epsilon_{\min }=2.1 \times 10^{-13} \mathrm{erg} \mathrm{cm}^{-3}\right)$, the minimum magnetic field $\left(B_{\min }=1.5 \times 10^{-6} \mathrm{G}\right)$, the minimum total pressure $\left(P_{\min }=1.1 \times 10^{-13}\right.$ dyne $\left.\mathrm{cm}^{-2}\right)$, and the degree of linear polarization $(m=20-40 \%)$.

The leptonic component of the CRs will produce synchrotron emission with a spectrum given by $S_{\nu} \propto \nu^{-\alpha}$, where $\alpha=(\Gamma-1) / 2$. Since $\alpha=0.6$, we get $\Gamma=2.2$. In what follows, we shall assume that electrons and protons in the source obey the same power law energy spectrum $\propto E^{-\Gamma}$.

The degree of linear polarization expected for the synchrotron radiation when the magnetic field is homogeneous is

$$
m_{0}(\Gamma)=\frac{3 \Gamma+3}{3 \Gamma+7} \approx 71 \%
$$

However, the observed degree of polarization has a mean value of $m(\Gamma) \sim 30 \%$. This fact can be explained by the presence of a turbulent component $B_{\mathrm{r}}$ in the field, in such a way that

$$
m(\Gamma)=m_{0}(\Gamma) \frac{B_{0}^{2}}{B_{0}^{2}+B_{\mathrm{r}}^{2}}
$$

where $B_{0}$ stands for the homogeneous field. From Eq. (2) and (3) we get

$$
B_{\mathrm{r}} \approx 1.2 B_{0}
$$

and consequently $u=B_{\mathrm{r}}^{2} / B_{\text {total }}^{2} \approx 0.6$.
The radius of the acceleration region can be directly measured by means of a Gaussian fitting from the detailed VLA maps obtained by Killeen et al. [5], resulting $R_{\mathrm{hs}} \approx 2.5 h^{-1} \mathrm{kpc}$. The velocity of the jet is not well established. If the source is $\sim 10^{7}$ yr old, a velocity $\beta_{\text {jet }} \sim 0.2$ can be estimated from an analysis of the energy budget [11]. The value of $A$ in Eq. (2) has been computed by Biermann and Strittmatter [10] almost independently of the source parameters. They obtain $A \approx 200$. A value $a \sim 0.01$ seems to be reasonable for a source with the luminosity of PKS 1333-33 [10]. Taking the above considerations into account (with a typical value for the total magnetic field in the spot of $B_{-5} \sim 10$ [12]), we obtain from Eq. (2) the maximun injection energy for protons, $E_{\max } \approx 6 \times 10^{20} \mathrm{eV}$.

The diffusive shock acceleration process in the working surface of the eastern lobe leads to a power law particle spectrum [13],

$$
Q(E)=\kappa E^{-\Gamma} \quad\left(E_{0}<E<E_{\max }\right)
$$

where $\kappa=\left(n_{0} / \Gamma-1\right) E_{0}^{1-\Gamma}$ for $E_{\max } \gg E_{0}$, and $n_{0}$ is the particle density in the source. Thus, we have the following proton injection spectrum

$$
Q(E) \propto E^{-2.2}, \quad E_{0}<E<6 \times 10^{20} \mathrm{eV} .
$$

Let us now estimate the losses that the high energy protons suffer during their journey through the intergalactic medium, and the consequent modification of the injection spectrum. This energy degradation originates in the interaction of the protons with the very low energy photons of the cosmic microwave background (CMB). For nearby sources (distances $<100 \mathrm{Mpc}$ ) photomeson production is the dominant interaction at $E>10^{19} \mathrm{eV}$ (if one considers the contribution coming from the tail of the Planck distribution) [14]. The propagation of the protons through the relic gas of photons may be modelled by a kinetic transport equation. Although it is clear that the interactions of the ultra high energy protons with the CMB photons lead to a step-by-step energy loss (which should be included in the transport equation as a collision integral), hereafter we shall use the continuous energy loss approximation assuming straight line propagation which is expected to be in agreement with the physics of the problem at hand.

Using the formalism presented in [14] it is straightforward to compute the main characteristic of the evolved spectrum. It is well known that the conservation of the differential particle number implies that,

$$
\frac{\partial N}{\partial t}=\frac{\partial(b(E) N)}{\partial E}+D \nabla^{2} N+Q
$$

where, in the first term on the right, $b(E)$ is the mean rate at which particles lose energy $(\approx 3.66 \times$ $10^{-8} E \exp \{287 / E\} E e V / y r$ [14]); the second term, the diffusion in the CMB, is found to be extremely small due to the low density of relic photons and is neglected in 


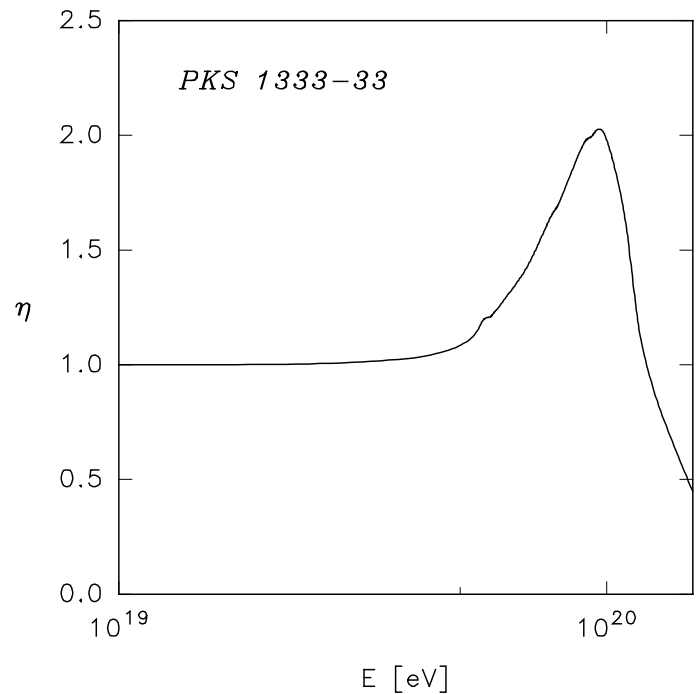

FIG. 2. Modification factor of PKS 1333-33.

the following. The third term corresponds to the particle injection rate into the intergalactic medium given by Eq. (6). The solution of Eq. (7) reads,

$$
N(E, t)=\kappa E_{g}^{-\Gamma} \frac{b\left(E_{g}\right)}{b(E)}
$$

with the injection energy $E_{g}$ fixed by the constraint,

$$
3.66 \times 10^{-8} t \mathrm{yr}^{-1}-\operatorname{Ei}(287 / E)+\operatorname{Ei}\left(287 / E_{g}\right)=0
$$

being $\operatorname{Ei}(x)$ the exponential-integral function. The evolution of the injection spectrum can be then conveniently represented by the modification factor $\eta$, defined as the ratio between the modified and the unmodified spectrum. In Fig. 2 we have plotted the relation obtained for $\eta$ in the case of PKS 1333-33. The energy loss by photomeson production creates the expected cutoff, and the resulting ultra high energy CRs (protons and neutrons) pile up just below the threshold energy of photopion production, forming a bump. However, the energies at which the cutoff and the bump appear seem to indicate that CRs above $100 \mathrm{EeV}$ might be expected from the location in the sky of PKS $1333-33\left(l \approx 313.7^{\circ}, b \approx 27.7^{\circ}\right)$.

The question whether this scenario is the correct one or not should be answered in a few years by the Pierre Auger Southern Observatory [15] (fluorescence detector plus ground array) as well as by the future eyes of the OWL [16] that will deeply watch into the CR-sky.

\section{ACKNOWLEDGMENTS}

We are indebted to N. E. B. Killeen, G. V. Bicknell, and R. D. Ekers for permission to reproduce the VLA image of PKS 1333-33. G. E. R. especially thanks the help by J. Blumina Romero. This work has been partially supported by FOMEC, CONICET, CLAF-CNPq, and the agency ANPCT.
[1] K. Greisen, Phys. Rev. Lett. 16, 748 (1966); G. T. Zatsepin and V. A. Kuz'min, Pis'ma Zh. Éksp. Teor. Fiz. 4, 114 (1966) [JETP Lett. 4, 78 (1966)].

[2] Actually, the already reported air shower events produced by particles with energies above $4 \times 10^{19} \mathrm{eV}$ exhibit a correlation with the general direction of the supergalactic plane, where a large number of nearby radio galaxies is located. T. Stanev, P. L. Biermann, J. Lloyd-Evans, J. P. Rachen and A. A. Watson, Phys. Rev. Lett. 73, 3056 (1995).

[3] G. Cavallo, Astron. Astrophys. 269, 45 (1978); G. E. Romero, J. A. Combi, S. E. Perez Bergliaffa, and L. A. Anchordoqui, Astropart. Phys. 5, 279 (1996). See also, M. Hillas, Nature 395, 15 (1998).

[4] J. P. Rachen and P. L. Biermann, Astron. Astrophys. 272, 161 (1993).

[5] N. E. B. Killeen, G. V. Bicknell, R. A. Ekers, Ap. J. 302, 306 (1986).

[6] O. B. Slee, Australian J. Phys. Suppl. 43,1 (1977).

[7] W. Freedman, in Proc. of the $18^{\text {th }}$ Texas Symposium on Relativistic Astrophysics and Cosmology, A. Olinto, J. Frieman and D. Schramm Eds. (World Scientific, Singapore, 1997).

[8] K. Meisenheimer et al., Astron. Astrophys. 219, 63 (1989).

[9] R. D. Blandford and M. J. Rees, Mon. Not. R. Astr. Soc. 169, 395 (1974).

[10] P. L. Biermann and P. A. Strittmatter, Ap. J. 322, 643 (1987).

[11] N. E. B. Killeen and G. V. Bicknell, Ap. J. 324, 198 (1988).

[12] P. L. Biermann, in American Institute of Physics Conference Proc. Observing the Highest Energy Particles from Space, December 1997; Max-Plank-Institut für Radioastronomie, preprint No. 739.

[13] A. R. Bell, Mon. Not. R. Astr. Soc. 182, 147 (1978).

[14] L. A. Anchordoqui, M. T. Dova, L. N. Epele, and J. D. Swain, Phys. Rev. D 55, 7356 (1997); Nucl. Phys. B (Proc. Suppl.) 52B, 249 (1997).

[15] The Auger Collaboration, Pierre Auger Project Design Report, Fermi National Accelerator Laboratory, available electronically from: http://www-td-auger.fnal.gov:82

[16] J. F. Ormes et al., in Proc. of the $25^{\text {th }}$ International Cosmic Ray Conference, (Durban, S.A.) M. S. Potgieter, B. C. Raubenheimer and D. J. van der Walt Eds. 5, 273 (1997). 